\title{
Ram-Pressure Induced Star Formation in the LMC
}

\author{
Chiara Mastropietro $^{\mathrm{A}, \mathrm{C}}$, Andreas Burkert ${ }^{\mathrm{A}}$, and Ben Moore ${ }^{\mathrm{B}}$ \\ A Universitäts Sternwarte München, Scheinerstr. 1, D-81679 München, Germany \\ B Institute for Theoretical Physics, University of Zürich, CH-8057 Zürich, Switzerland \\ C Corresponding author. Email: chiara@usm.uni-muenchen.de
}

Received 2007 November 15, accepted 2008 April 23

\begin{abstract}
We use high-resolution n-body/SPH simulations to study the hydrodynamical interaction between the Large Magellanic Cloud and the hot halo of the Milky Way. We investigate whether the ram-pressure acting on the gaseous disk of the satellite can explain the peculiarities observed in the HI distribution and the location of the recent star formation activity.
\end{abstract}

Keywords: galaxies: Magellanic Clouds — methods: n-body simulations

\section{Introduction}

The Large Magellanic Cloud (LMC) has revealed a very complex structure both in the stellar and in the gaseous component. The elongation of the stellar disk in the direction of the Galactic center, its substantial vertical thickness, the warp and the strong asymmetric bar are naturally predicted by numerical simulations as a result of the gravitational interaction between the LMC and the Galaxy (Bekki \& Chiba 2005; Mastropietro et al. 2005). The old stellar distribution appears to be quite smooth in the outer parts of the disk, with no signs of spiral structures out to a radius of $10 \mathrm{kpc}$. Within the same radius the $\mathrm{HI}$ large scale structure reveals the presence of several asymmetric features which do not have an equivalent in the old stellar disk. The gaseous disk is limb-brightened, with a secondary peak in the surface density profile at large radius, and characterized by the presence of an elongated region localized at the south-east of the galaxy and aligned with the border of the optical disk, where the column density distribution shows a steep increase (Staveley-Smith et al. 2003).

Since the LMC proper-motion vector is directed to the east, it appears reasonable to associate this high-density region to the ram-pressure acting on the leading edge of the disk due to the orbital motion of the LMC and its consequent interaction with the diffuse hot gas in the halo of the Milky Way (MW). The presence of an extended hot halo surrounding galaxies and in hydrostatic equilibrium within the dark matter potential is expected by current models of hierarchical structure formation. A symmetric soft X-ray emission has been recently detected around the massive galaxy NGC5746 out to at least $20 \mathrm{kpc}$ from the disk (Pedersen et al. 2006). Indirect observations have confirmed the existence of a extended distribution of hot ( $T \sim 10^{6} \mathrm{~K}$ ) gas also in the MW (Sembach et al. 2003). Constraints from dynamical and thermal arguments fix the density of the gaseous halo in a range between $10^{-5}$ and $10^{-4} \mathrm{~cm}^{-3}$ at the LMC distance from the Galactic center.
Cioni et al. (2006) have recently performed a detailed analysis of the LMC global star-formation rate using asymptotic giant branch stars, which trace the intermediate age population in the field. They find an irregular and patchy distribution in age, with the youngest carbon-rich systems localized at the south-east of the disk. The present star formation activity is rather clumpy and concentrated in stellar complexes characterized by intense HII emission and associated with bright $\mathrm{H} \alpha$ filamentary bubbles. Most of these very young structures lie in the south-east of the disk, in the proximity of 30 Doradus, the largest star forming region of the LMC, some are located in the bar and the remainder form an asymmetric pattern which covers the entire disk with no apparent relation to the global geometry of the satellite. It is not clear which is the overall physical mechanism responsible for triggering star formation and different models has been proposed in the past. The stochastic self-propagating star formation (SSPSF) model (Gerola \& Seiden 1978) predicts a clear age gradient in the LMC's stellar complexes, with the edges being younger respect to the center, in contradiction with observations (Braun et al. 1997). de Boer et al. (1998) proposed a scenario where the bow shock originated by the motion of the LMC through the hot galactic halo compresses the leading edge of the disk and induces star formation. The pressure at the south-eastern edge of the LMC is indeed 10 times higher than the average in the rest of the LMC (Blondiau et al. 1997). This model, which assumes the orbital motion vector lying in plane of the disk, predicts increasing ages of the stellar complexes in the direction of the rotation, due to the fact that the material compressed at the front side of the disk moves, in time, away to the side. The youngest systems would indeed lie at the south-east border of the disk, where the relative velocity between the corotating interstellar medium and the external diffuse gas is maximum. Several giant structures along the outer east and north edge of the LMC actually show a progression 
in age in a clockwise direction: moving from south-east to the north LMC 2, 30 Doradus and LMC 3, LMC 4, NGC1818.

Grebel \& Brandner (1998) studied the recent star formation history of the LMC using cepheids and other supergiant stars and found that although the majority of the star formation events in the last $30 \mathrm{Myr}$ are concentrated on the east border, others are distributed across the entire disk in partial contrast with the bow shock induced star formation model, which can not explain them. Another approach explains the present spatial distribution of young stars with a close interaction between the Magellanic Clouds 20 Myr ago (Bekki \& Chiba 2007). Such an interaction, which would also be responsible for the formation of the LMC bar and the asymmetric Hi spiral arms, should induce strong perturbations in the old stellar disk, in contrast with observations.

The aim of this paper is to use high resolution $\mathrm{SPH}$ simulations to study the effects of the interaction between the LMC interstellar medium and the diffuse hot halo of the MW. We want to investigate whether the ram-pressure acting on the leading edge of the LMC disk is responsible for the increase in density observed in the south-east and for triggering star formation. The analytic model of de Boer et al. (1998) assumes a pure edge-on model, but according to van der Marel et al. (2002) the present angle between the LMC disk and the orbital motion is nearly $30^{\circ}$. Even in the absence of precession and nutation, this angle is subjected to large variations during the orbital period. Moreover, the ram-pressure felt by the LMC is not constant and has a maximum when the satellite approaches a perigalacticon passage. The motion of the LMC through the hot halo of the MW from an apogalacticon passage to the last perigalacticon is modeled using 'test wind tunnel' simulations with increasing ram-pressure values.

\section{Models}

The initial conditions of the simulations are constructed using the technique described by Hernquist (1993). Our disk galaxy model is multi-component system with a stellar and gaseous disk embedded in a spherical NFW dark matter halo. The density profile of the dark halo is adiabatically contracted in response to baryonic infall. The stellar disk follows an exponential surface density profile while the gaseous disk is characterized by an exponential profile with the same radial and vertical scale length as the stellar component and by a constant density layer which extends up to eight scale radii. The structural parameters of the disk and the halo are chosen so that the resulting rotation curve resembles that of a typical bulgeless late-type $(\mathrm{Sc} / \mathrm{Sd})$ disk galaxy. They are similar to those adopted in M05 for the initial LMC model and reproduce quite well the peak of the rotation curve inferred by van der Marel et al. (2002).

As seen in M05, the interaction with the MW does not affect significantly the stellar and dark matter mass in the inner 8-9 kpc of the LMC and consequently the global rotation curve within this radial range. The choice of an extended gaseous component for the initial LMC model is

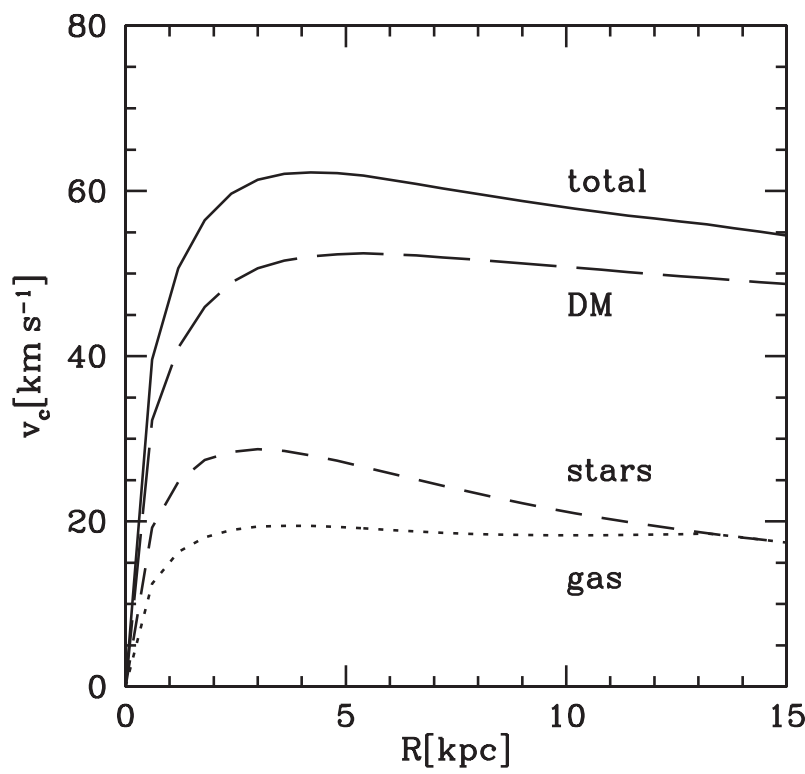

Figure 1 Galaxy model rotation curves.

motivated by the fact that spiral galaxies in the local universe are commonly observed to be embedded in extended disks of neutral hydrogen significantly larger than their stellar component. The combined effect of gravity and ram-pressure stripping can remove a significant fraction of gas from a disky MW satellite with orbital and structural parameters comparable to those of the LMC(M05). In support of this model, Bland-Hawthorn et al. (2007) have now shown that the previously unexplained $\mathrm{H}$-alpha emission along the Magellanic Stream is consistent with the stripped gas breaking up after interacting with the hot Galactic halo. Their model parameters are essentially identical to those used in the present work.

For consistency, we must account for the stripped gas at the start of the simulation. After less than two orbital periods ( $\sim 4 \mathrm{Gyr})$ the ram-pressure stripping radius is a factor of three smaller than the initial radius of the gaseous disk. In the present work we neglect the presence of gravitational forces focusing on the effects of pure ram-pressure. Therefore we do not expect to see a significant decrease in the radius of the gas distribution. However in order to take into account the loss of cold gas from the disk of the satellite and the star formation events, we assumed an initial amount of gas in the disk which is $\sim 2-3$ times larger than the HI mass in the LMC.

The mass within the virial radius was set equal to $2.18 \times 10^{10} \mathrm{M}_{\odot}$ and the fraction of mass in the disk is $\sim 10 \%$, equally distributed between the gaseous and stellar component. The contribution of the different components to the global rotation curve, assuming a disk scale length $R_{\mathrm{d}}=1.7 \mathrm{kpc}$ and a concentration $c=9.5$ is plotted in Figure 1 . The halo spin parameter, which sets the disk scale length in our modeling, is $\lambda=0.074$.

The initial stellar disk of the satellite galaxy has, within the scale radius, a central mass surface density of $\sim 35 \mathrm{M}_{\odot} \mathrm{pc}^{-2}$ (Figure 2), which corresponds to a B-band 


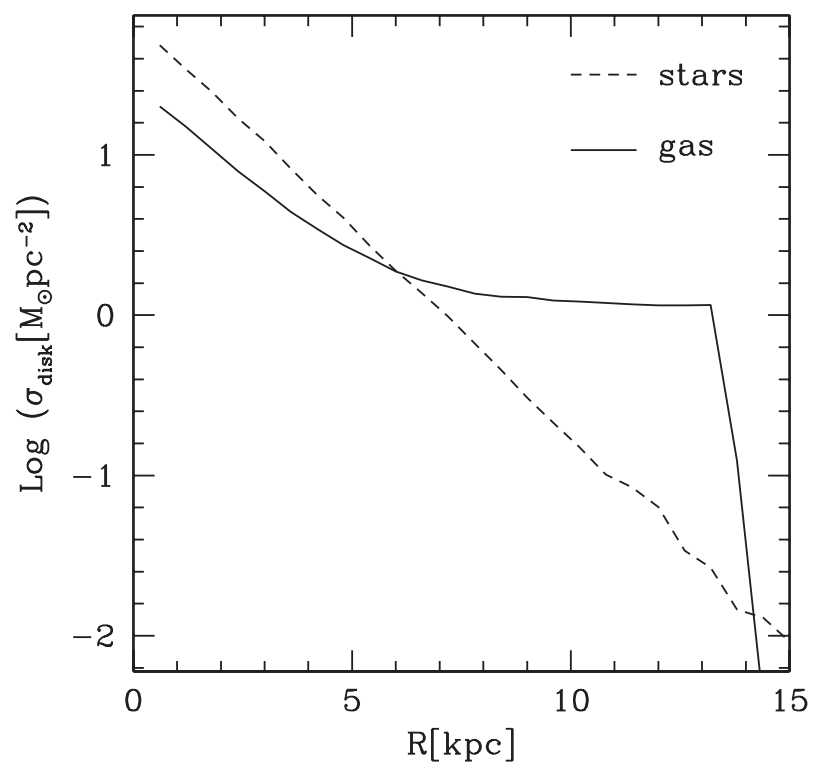

Figure 2 Gaseous and stellar disk surface density profiles.

surface brightness of $\sim 24 \mathrm{mag} \operatorname{arcsec}^{-2}$, assuming a mass to light ratio $\simeq 2$. The central gas surface density is only $\sim 16 \mathrm{M}_{\odot} \mathrm{pc}^{-2}$ since a significant fraction of gas is distributed in the external disk. Assuming 72\% HI fraction this value corresponds to an hydrogen column density of $\sim 1.5 \times 10^{21} \mathrm{~cm}^{-2}$ within $R_{\mathrm{d}}$, which is comparable with the values observed by Staveley-Smith et al. (2003) with the LMC Parkes multibeam Hi survey.

In order to obtain a strongly stable disk against bar formation even in the presence of significant gas stripping and consequent perturbation of the satellite potential, the Toomre (Toomre 1964) stability criterion is largely satisfied and the disk initially evolved in isolation for 1 Gyr.

All the simulations in this work were carried out using GASOLINE, a parallel tree-code with multi-stepping (Wadsley, Stadel \& Quinn 2004). The code includes radiative cooling for a primordial mixture of hydrogen and helium in collisional equilibrium. We used a star formation recipe which includes density and temperature criteria, while converging flow (Katz 1992) is not required in most of the simulations due to the particular geometry of the system. Gas particle are eligible to form stars only if the star formation region has a minimum physical density corresponding to 0.1 hydrogen atoms per $\mathrm{cm}^{3}$ and an overdensity $\rho_{\text {gas }} / \overline{\rho_{\text {gas }}}>55.7$ (Katz, Weinberg \& Hernquist 1996) (hereafter KWH), which basically restricts star formation to collapsed, virialized regions. The star-formation rate is assumed to be proportional to $\rho_{\text {gas }}^{3 / 2}$, where $\rho_{\text {gas }}$ represents the volume density of the cold gas, and is given by the expression (Katz et al. 1996)

$$
\frac{\mathrm{d} \rho_{\star}}{\mathrm{d} t}=\frac{c_{\star} \rho_{\mathrm{gas}}}{t_{\mathrm{form}}}
$$

where the star formation timescale $t_{\text {form }}$ is the maximum between the local gas dynamical time $t_{\text {dyn }}=$ $\left(4 \pi G \rho_{\text {gas }}\right)^{-1 / 2}$ and the local cooling time. If the gas is already cool enough to form stars i.e $T<T_{\max }$, then $t_{\text {dyn }}$ is used. We assumed $T_{\max }=30000 \mathrm{~K}$. The constant starformation rate parameter $c_{\star}$ is chosen in order to reproduce the global LMC star-formation rate. The mass of the newly formed collisionless particle is a fixed fraction $\epsilon$ of the parent gas particle, whose mass is reduced accordingly. Following Katz (1992) we assumed for our favourite models a star formation efficiency $\epsilon$ equal to one third.

In order to study the influence of pure ram pressure on a galaxy model orbiting in a Milky Way halo, we performed 'wind tunnel' runs where the ram-pressure value varies with time and simulated the last 1 Gyr of the LMC's orbit.

We represent the hot gas as a flux of particles moving along the major axis of an oblong of base equal to the diameter of the dark matter halo of the galaxy and height $h=v t$, where $v$ is the velocity of the LMC at the perigalacticon and $t$ is set equal to the orbital period of the satellite. The hot particles have an initial random distribution and a temperature $T=10^{6} \mathrm{~K}$. The box has periodic boundary conditions in order to restore the flow of hot gas that leaves the oblong. The disk galaxy model is at rest at the center of the oblong. Kinematical data (van der Marel et al. 2002; Kallivayalil et al. 2006) suggest that the LMC is presently just past a perigalactic passage. The largest ram-pressure on the LMC's ICM is expected during the last orbital semiperiod due to the increasing velocity and external pressure acting on the satellite. The external pressure felt by the disk in the wind tube varies from $P_{\min }$ at the apogalacticon to $P_{\max }$ at the point of closest approach to the Galactic center. The hot gas densities are comparable with those provided by M05, who modeled the MW gaseous halo assuming a spherical distribution of gas which traces the dark matter profile, with a mean number density of $2 \times 10^{-5} \mathrm{~cm}^{-3}$ within $150 \mathrm{kpc}$. The orbital parameters are the same as in M05 for most of the runs (with a maximum velocity $v_{\max }=250 \mathrm{~km} \mathrm{~s}^{-1}$ ), but models with a higher pericentric velocity are also explored, according to the last proper motion measurements of Kallivayalil et al. (2006), as well as models with a Galactic halo characterized by a lower gas density.

Each galaxy model is simulated using $7 \times 10^{5}$ particles, of which $6 \times 10^{5}$ are in the dark halo and $1.5 \times 10^{5}$ in the disk $\left(10^{5}\right.$ collision and $5 \times 10^{4}$ collisionless $)$. The hot gas in the 'wind tunnel' has $2 \times 10^{6}$ particles. The gravitational spline softening is set equal to $0.5 \mathrm{kpc}$ for the dark halo and the hot gas in the oblong, while it is $0.2 \mathrm{kpc}$ for stars and gas in the disk.

\section{Cooling Simulations}

In order to study the effect of pure compression on the density distribution of cold gas in the LMC disk, we run a first set of simulations where the gas cools radiatively but star formation is not activated.

The present angle between the LMC disk and its proper motion vector is roughly $30^{\circ}$. Even neglecting the effects of precession and nutation on the disk plane of the satellite this angle is expected to vary significantly during an orbital 
period, especially in the proximity of a pericentric passage, due to the rapid changes in the velocity vector. Different relative orientations of the LMC disk with respect to the orbital motion are therefore investigated. The inclination angle $i$ is defined as the angle between the angular momentum of the disk and the flux of hot particles in the wind tube, so that a galaxy moving edge-on through the external medium is characterized by $i=90^{\circ}$ (run cool90), while the present LMC disk would have $i \sim 60^{\circ}$. We also explored models with an inclination angle $i=45^{\circ}$ and $10^{\circ}$ (runs cool45 and cool10, respectively). The velocity at the pericenter is $250 \mathrm{~km} \mathrm{~s}^{-1}$.

Figures 3-5 illustrate the changes in the disk gas density distribution as the satellite passes through increasing values of the external pressure moving from the apogalacticon to the next perigalacticon. In all the three figures which refer to different orientations of the satellite - each couple of panels illustrates the state of the disk at increasing (from the top to the bottom) times along the simulated orbit: $t=0.4,0.6,0.8$ and $1 \mathrm{Gyr}$. The series of four panels on the left represents the Hi column density maps. The density contrast is chosen in such a way to evidence the density gradient in the external disk, since the gas distribution in the central regions of the LMC is dominated by the presence of the bar and a direct comparison with these simulations is not possible. Therefore we studied in detail the behaviour of gas between 7 and $15 \mathrm{kpc}$ from the center. The hot gas particles flow on to the disk from the left to the right of each plot, with increasing ram-pressure values moving from the first to the fourth image. The disk is seen face-on and is rotating clockwise. In the case of the edgeon run (Figure 3 ) cold gas particles lying in the left-bottom quadrant of the disk feel the largest ram-pressure due to the fact that their relative velocity with respect to the external medium is maximum. The four panels on the right represent the change in the mean volume density and radius of the gaseous external disk as a function of the azimuthal angle $\phi$. Referring to the geometry of the Hi density images on the left, $\phi=0$ corresponds to the bottom of the disk and increases clockwise in such a way that the disk moves in the direction of $\phi=90^{\circ}$. The initial azimuthal profiles (not represented in the plots) are flat since both these quantities have only radial dependence. As soon as the satellite starts moving through the outer medium the external gas density develops a peak centered on $\sim \phi=90^{\circ}$ : disk particles localized in regions of maximum ram-pressure get compressed and move on inner orbits, while their circular velocity increases consequently. After about a quarter of orbital period the gas has reached its minimum radius and maximum local density. The gaseous disk becomes strongly asymmetric: the compression of the front edge produces a density increase along the left border of the disk, evident in the HI maps even at early times. The high density region forms a thin $(\sim 1.5 \mathrm{kpc})$ but continuous and well defined arc. At the perigalacticon it extends for almost $180^{\circ}$ like a spiral arm which has not an equivalent in the stellar distribution. Behind this feature the density of the cold gas drops drastically of more than one order of magnitude. In the case of a satellite moving through the hot medium with an inclination angle different from $90^{\circ}$ the external pressure directed perpendicularly to the plane of the disk increases as cos $i$ (Roediger \& Brüggen 2006) while the compression on the leading edge is much less pronounced. Figures 4 and 5 refer to runs with inclination angles $i=45^{\circ}$ and $i=10^{\circ}$. The disks are shown face-on. The increment in density along the leading edge is slower respect to the edge-on model (cool45) either nearly absent (cool10), while the compression in the direction perpendicular to the plane of the disk produces local gravitational instabilities in the external gaseous disk (see also Mayer, Mastropietro \& Tran in preparation). This effect is more evident in run cool10 (almost face-on), where high density filaments delimitate regions where the local density is almost one order of magnitude lower. Despite the absence of a peak in the mean density azimuthal profile, the integrated final density of cool10 is comparable with the other runs.

Figure 6 represents the azimuthally averaged Hi column density profile of the final disk for the three different runs. As a result of the increase in density along the edge of the disk, the mean column density appears to be limbbrightened. The external profiles of cool90 and cool45 are very similar, while in the run cool10 the secondary peak is located at larger radius.

\section{Simulations with Star Formation}

The main characteristics of the different star formation runs are summarized in Table 1 . In Table 1 the second column indicates the use of the converging flow criterion in the star formation recipe, the third and the fourth columns represent the star formation efficiency $\epsilon$ and the star-formation rate parameter $c_{\star}$, respectively, while $i$ is the inclination angle of the disk i.e. the angle between the angular momentum of the disk and the flux of hot particles. A model with $i=90$ is indeed a disk moving edge-on in the external medium. The sixth and seventh columns represent the minimum and maximum ram pressure suffered by the disk during a semi-orbital period. The last column indicates the presence of a temperature floor.

Our standard star formation model does not include the converging flow criterion and is characterized by an efficiency $\epsilon=0.33$ (Katz 1992). The star-formation rate parameter $c_{\star}$ is initially set equal to 0.02 . This model was adopted to run wind tube simulations with inclination angles $i=90,45,10^{\circ}$ (SF90, SF45, SF10). The orbital parameters are the same as in M05, with a perigalactic velocity of $250 \mathrm{~km} \mathrm{~s}^{-1}$. We also investigated different star formation recipes requiring converging flow and assuming different values of $c_{\star}$ and $\epsilon$. We explored star-formation rate parameter values in the range from 0.01 to 0.05 , which produce a star-formation rate integrated over the entire disk comparable with the $0.1 \mathrm{M}_{\odot} \mathrm{yr}^{-1}$ provided by Sandage (1986). An efficiency $\epsilon=1$ implies that whenever a gas particle satisfies the star formation requirements, it is immediately turned into a single particle of the same mass. 

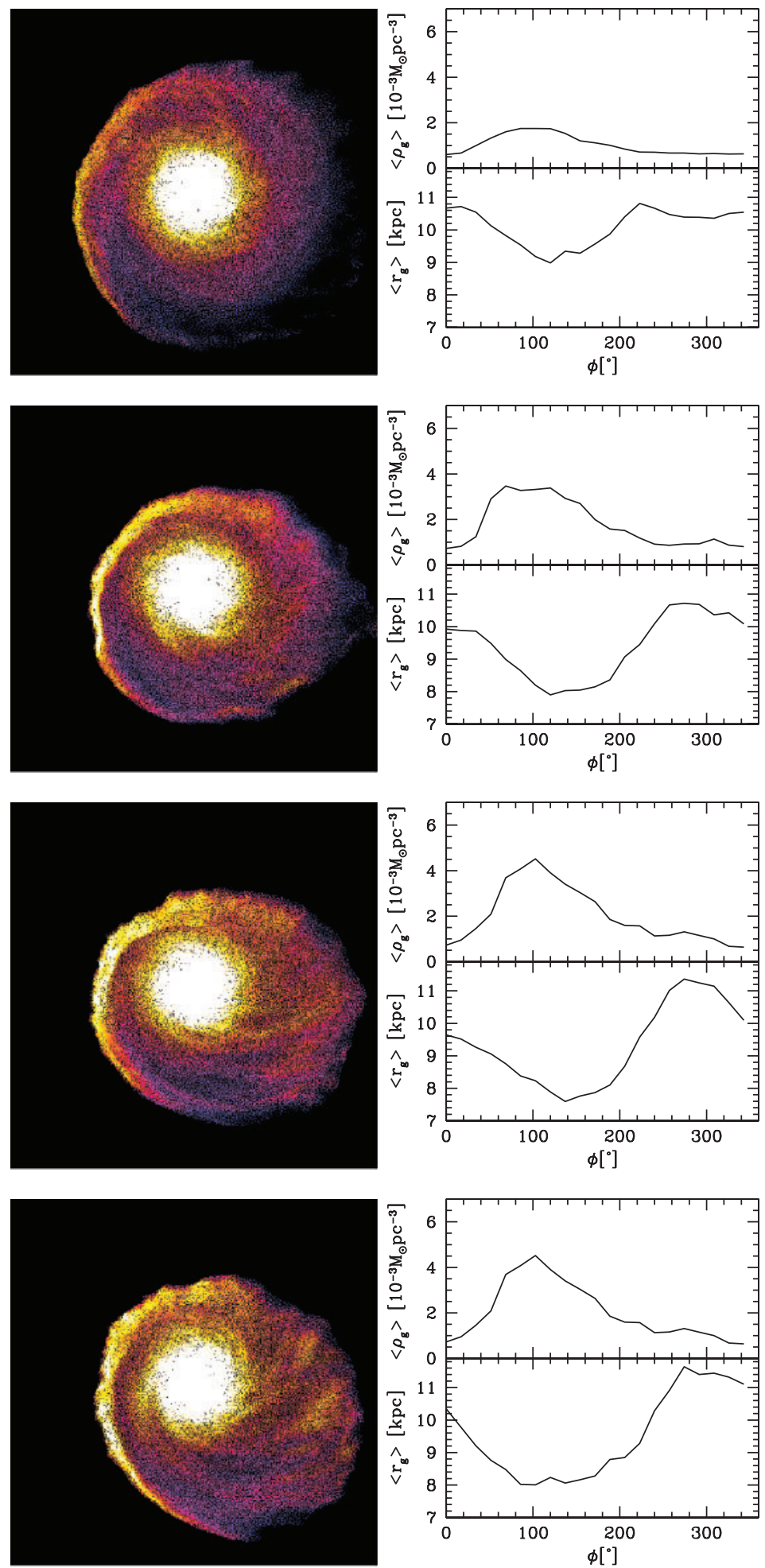

Figure 3 Edge-on model (run cool90). Evolution of the gas density distribution due to ram-pressure forces imposed by an external hot halo. Left panels: Hi column density maps (the satellite is moving towards the left of the page). Colours represent a logarithmic scale where white corresponds to a density larger than $1.2 \times 10^{21} \mathrm{~cm}^{-2}$ and blue to values lower than $5 \times 10^{19} \mathrm{~cm}^{-2}$. Right panels: mean gas density and radius of the external disk as functions of the azimuthal angle $\phi$. Each couple of plots (from the top to the bottom) represents the state of the disk at increasing times along the orbit: $t=0.4,0.6,0.8$ and $1 \mathrm{Gyr}$.

In the last six simulations listed in Table 1 we used the standard star formation model to investigate the effects of different orbital parameters and gas halo densities. Runs SFv400 are characterized by a maximum ram pressure value which corresponds to a perigalactic velocity of $400 \mathrm{~km} \mathrm{~s}^{-1}$ (Kallivayalil et al. 2006), assuming that the density profile of the hot halo remains unchanged. In order to reduce gravitational instabilities and fragmentation in a 

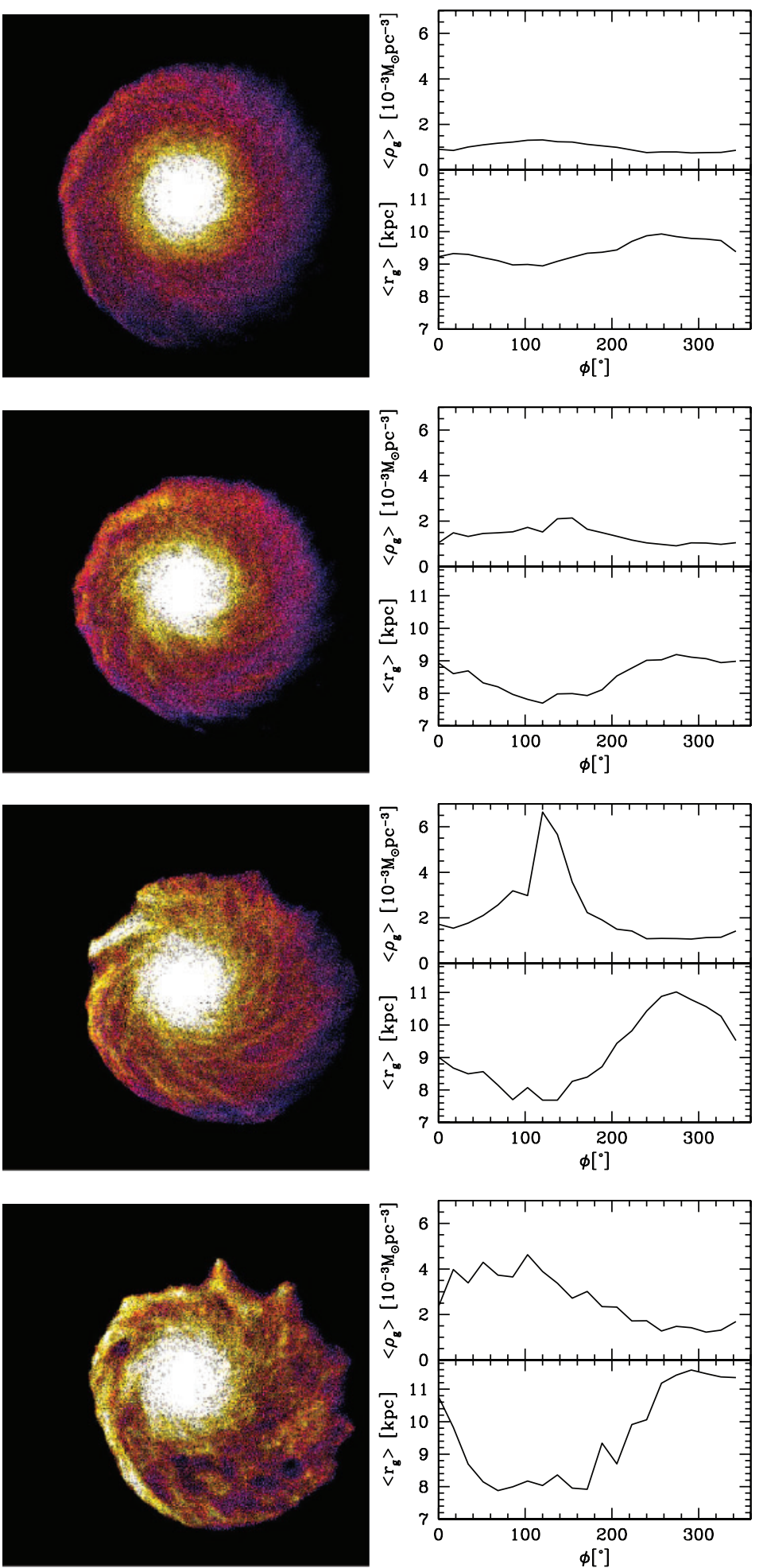

Figure 4 Model with an inclination angle $i=45^{\circ}$ (run cool45). Evolution of the gas density distribution due to ram-pressure forces imposed by an external hot halo. Left panels: Hi column density maps (the satellite is moving towards the left of the page). Colours represent a logarithmic scale where white corresponds to a density larger than $1.2 \times 10^{21} \mathrm{~cm}^{-2}$ and blue to values lower than $5 \times 10^{19} \mathrm{~cm}^{-2}$. Right panels: mean gas density and radius of the external disk as functions of the azimuthal angle $\phi$. Each couple of plots (from the top to the bottom) represents the state of the disk at increasing times along the orbit: $t=0.4,0.6,0.8$ and $1 \mathrm{Gyr}$.

gaseous disk affected almost perpendicularly by stronger ram-pressure values (SF10v400), we introduced temperature floors of 12000 and $15000 \mathrm{~K}$ (SF10v400t12000 and SF10v400t15000, respectively). SF90v400 is the corresponding edge-on run, simulated without thresholds in temperature, since edge-on models are not affected by local instabilities even in the presence of a large external pressure. Finally, with simulations SF901d and SF10ld we 
also consider the possibility of a Galactic hot halo which is ten times less dense than our standard model.

Figure 7 illustrates the state of the newly formed stellar disk at increasing times along the satellite orbit: $t=0.4,0.6,0.8$ and $1 \mathrm{Gyr}$. Each couple of rows (from the top to the bottom) is associated with a run characterized by a different inclination angle. The first row of the couple represents the face-on projection of the disk with the
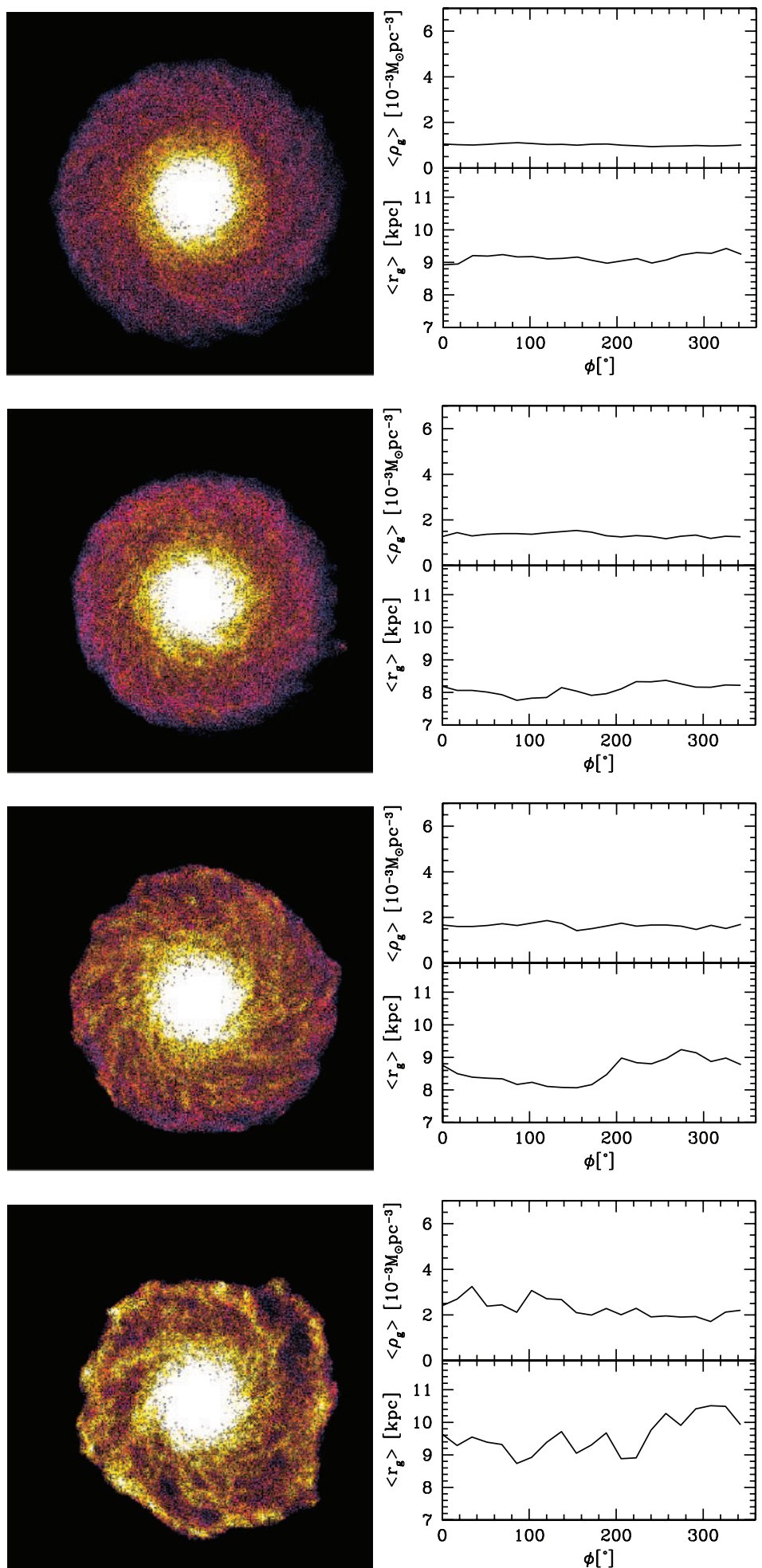

Figure 5 Model with an inclination angle $i=10^{\circ}$ (run cool10). Evolution of the gas density distribution due to ram-pressure forces imposed by an external hot halo. Left panels: Hi column density maps (the satellite is moving towards the left of the page). Colours represent a logarithmic scale where white corresponds to a density larger than $1.2 \times 10^{21} \mathrm{~cm}^{-2}$ and blue to values lower than $5 \times 10^{19} \mathrm{~cm}^{-2}$. Right panels: mean gas density and radius of the external disk as functions of the azimuthal angle $\phi$. Each couple of plots (from the top to the bottom) represents the state of the disk at increasing times along the orbit: $t=0.4,0.6,0.8$ and $1 \mathrm{Gyr}$. 
galaxy moving towards the left of the page and the same geometry of Figure 3. Time increases from the left to the right.

Each small cross indicates a new star formation event at the time of the snapshot (within a time interval of $40 \mathrm{Myr}$ ) while the circle delimitates the external disk $(r>7 \mathrm{kpc})$. The star formation activity in the inner disk is not represented. The second row represents the total mass $M_{\star}$ of the newly formed stars versus the disk azimuthal angle $\phi$. In the case of a galaxy moving edge-on through the external medium (SF90) stars form at the leading edge of the disk when the ram-pressure becomes larger than $\sim 5.9 \times 10^{-14} \mathrm{dyn} \mathrm{cm}^{-2}$, at $t \sim 0.3$ Gyr. The location of

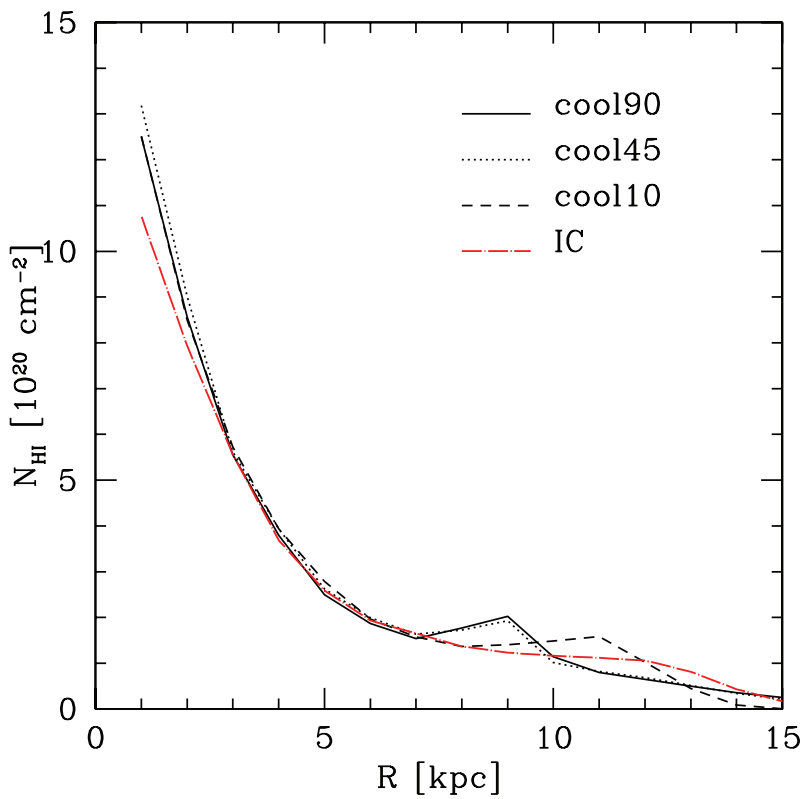

Figure 6 Azimuthally averaged Hi column density profile of the final LMC disk in units of $10^{20} \mathrm{~cm}^{-2}$. The curves refer to different runs. The red long-dashed line refers to the column density profile of the initial gaseous disk. the star formation events initially corresponds to the $\mathrm{HI}_{\mathrm{I}}$ column density peak observed in cool 190 around $100^{\circ}$ (Figure 3). Later on it expands along the entire front edge of the disk, creating a thin stellar arc well distinct from the star formation events which characterize the central disk. $M_{\star}$ shows a drastic drop at $\phi=200^{\circ}$. Runs with inclination $i<90^{\circ}$ are characterized by significant star formation only for values of the external pressure larger than $\sim 1.5 \times 10^{-13} \mathrm{dyn}^{-2}$. Panels referring to the nearly face-on run SF10 show that at $t>0.7 \mathrm{Gyr}$ star formation interest the entire external disk since the ram pressure affects the plane of the disk almost perpendicularly. Stars form along the delocalized and filamentary high density structures visible in Figure 5. The new star formation events appear to be distributed nearly homogeneously on the azimuthal profile of the external disk, although a the small peak in $M_{\star}$ is observable near $i=90^{\circ}$ due to the fact that the orientation of the disk respect to its orbital motion is not exactly face-on. The case of the intermediate run SF45 is more complex. In a first phase, for low values of the ram-pressure, star formation is determined by the compression at the leading edge and a thin star formation front appears on the east side of the disk, although not so well defined as in the case of a pure edge-on model. As soon as the external pressure reaches a critical level, the compression directed perpendicularly to the disk becomes the dominant mechanism which drives star formation.

Figure 8 illustrates the star-formation rate of the external disk. The three black curves refer to the standard star formation models. The edge-on disk starts forming stars earlier, but for large ram pressure values the star-formation rate of SF10 and SF45 grows much faster. At the perigalacticon passage SF45 has indeed a higher star-formation rate with respect to SF90. The remaining curves in Figure 4 refer to different star formation recipes (rows 4-9 of Table 1). The location of the star formation events in the external regions of the disk does not change significantly choosing different parameters in the star formation

Table 1. Star-formation simulations

\begin{tabular}{|c|c|c|c|c|c|c|c|}
\hline Run & Conv & $\epsilon$ & $c_{\star}$ & $\begin{array}{c}i \\
\text { (degrees) }\end{array}$ & $\begin{array}{c}P_{\min } \\
\left(10^{-13} \mathrm{dyn} \mathrm{cm}^{-2}\right)\end{array}$ & 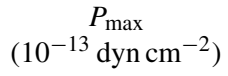 & $\begin{array}{c}T_{\min } \\
(\mathrm{K})\end{array}$ \\
\hline SF90 & No & 0.33 & 0.02 & 90 & $6.77 \times 10^{-2}$ & 2.11 & 0 \\
\hline SF45 & No & 0.33 & 0.02 & 45 & $6.77 \times 10^{-2}$ & 2.11 & 0 \\
\hline SF10 & No & 0.33 & 0.02 & 10 & $6.77 \times 10^{-2}$ & 2.11 & 0 \\
\hline SFconv & Yes & 0.33 & 0.02 & 90 & $6.77 \times 10^{-2}$ & 2.11 & 0 \\
\hline $\mathrm{SF} \in 1$ & No & 1 & 0.02 & 90 & $6.77 \times 10^{-2}$ & 2.11 & 0 \\
\hline SF90c0.01 & No & 0.33 & 0.01 & 90 & $6.77 \times 10^{-2}$ & 2.11 & 0 \\
\hline SF10c0.01 & No & 0.33 & 0.01 & 90 & $6.77 \times 10^{-2}$ & 2.11 & 0 \\
\hline SF90c0.05 & No & 0.33 & 0.05 & 90 & $6.77 \times 10^{-2}$ & 2.11 & 0 \\
\hline SF10c0.05 & No & 0.33 & 0.05 & 90 & $6.77 \times 10^{-2}$ & 2.11 & 0 \\
\hline SF90v400 & No & 0.33 & 0.02 & 90 & $6.77 \times 10^{-2}$ & 3.75 & 0 \\
\hline SF10v400 & No & 0.33 & 0.02 & 10 & $6.77 \times 10^{-2}$ & 3.75 & 0 \\
\hline SF10v400t12000 & No & 0.33 & 0.02 & 10 & $6.77 \times 10^{-2}$ & 3.75 & $1.2 \times 10^{4}$ \\
\hline SFISF10v400t15000 & No & 0.33 & 0.02 & 10 & $6.77 \times 10^{-2}$ & 3.75 & $1.5 \times 10^{4}$ \\
\hline SFd90 & No & 0.33 & 0.02 & 90 & $6.77 \times 10^{-3}$ & $2.11 \times 10^{-1}$ & 0 \\
\hline SFld10 & No & 0.33 & 0.02 & 10 & $6.77 \times 10^{-3}$ & $2.11 \times 10^{-1}$ & 0 \\
\hline
\end{tabular}



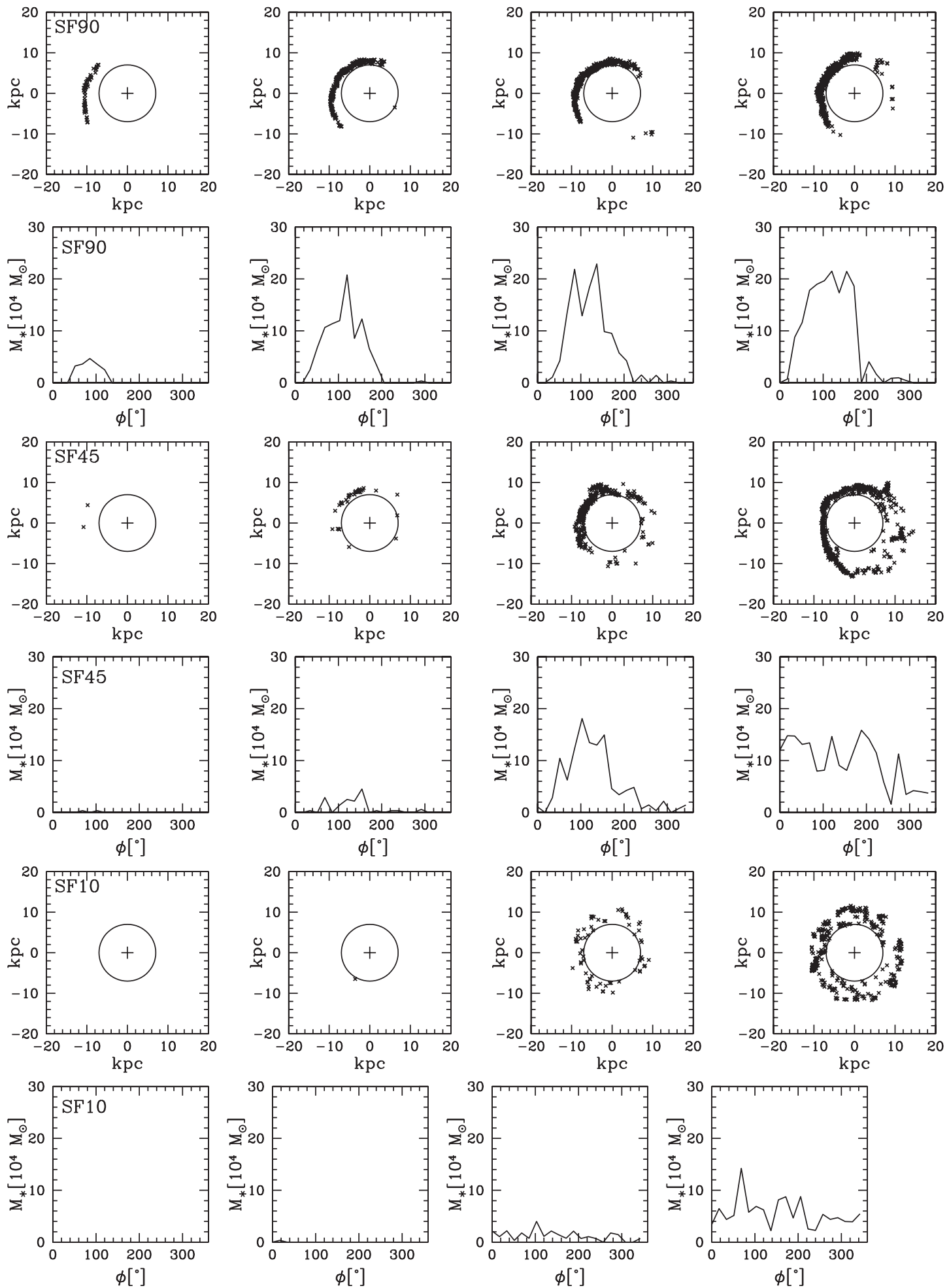

Figure 7 Location of the instantaneous star formation events in the external disk assuming different inclination angles. Each couple of rows corresponds to one of the first three runs of Table 1 and each panel to increasing times along the orbit: from the left to the right: $t=0.4,0.6,0.8,1 \mathrm{Gyr}$. The first row of the couple represents the disk seen in a face on projection, the second one the mass of the new stars versus the azimuthal angle $\phi$.

algorithm. The star-formation rate parameter $c_{\star}$ determines the amount of new stars forming but it does not affect the minimum threshold in ram-pressure neither the evolution of the star-formation rate. In particular in the case of edge-on runs after an initial steep increment the curve seems to converge to a constant value for increasing external pressures. The consequences of an increased star formation efficiency $(\mathrm{SF} \in 1)$ are almost negligible 


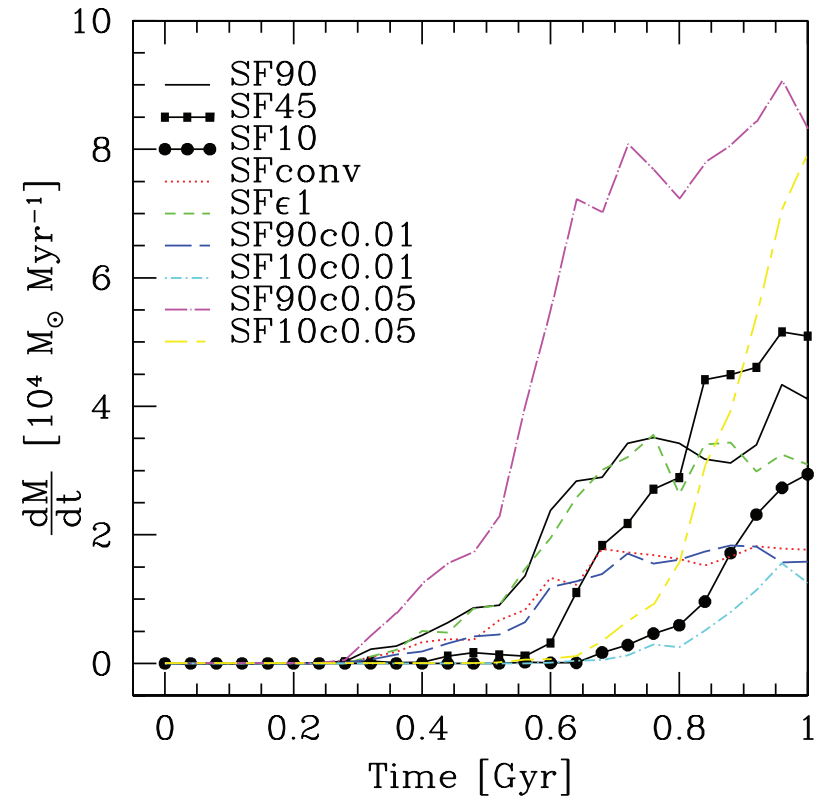

Figure 8 Star-formation rate of the disk as function of the azimuthal angle $\phi$ for the first nine runs listed in Table 1. Only the star formation events in the external disk $(r>7 \mathrm{kpc})$ are considered.

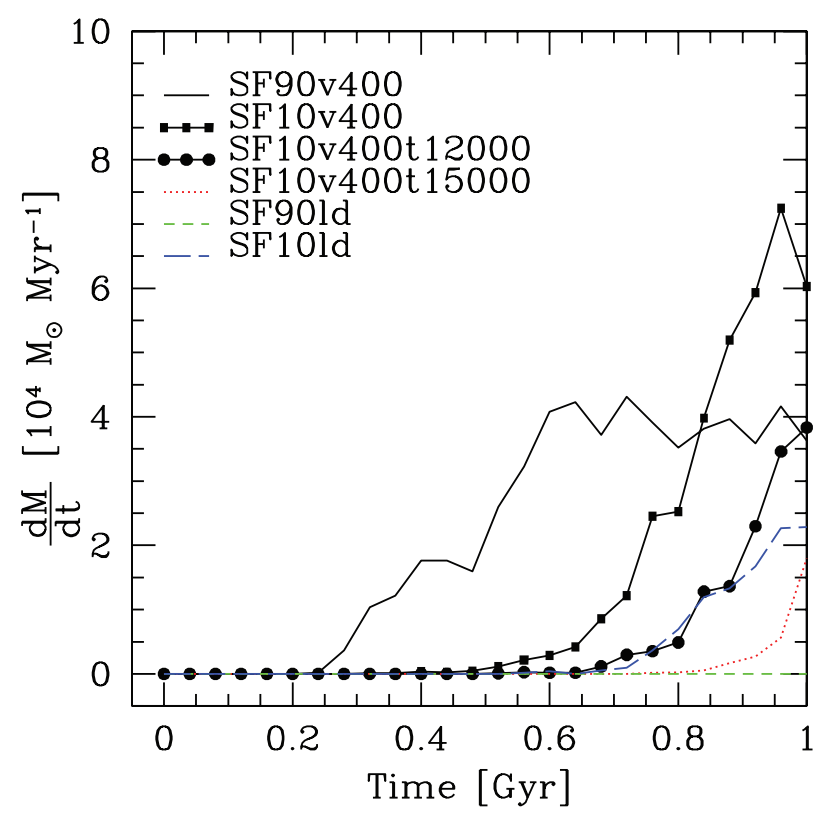

Figure 9 Star-formation rate of the disk as function of the azimuthal angle $\phi$ for the last six runs listed in Table 1. Only the star formation events in the external disk $(r>7 \mathrm{kpc})$ are considered.

while including the convergency requirement (SFconv) has nearly the same effect than to reduce the star-formation rate parameter of a factor two.

For convenience the star-formation rate of the last six runs of Table 1 is plotted separately (Figure 9). An edge-on model with larger orbital velocity (SF90v400) is characterized by a steeper increment in star formation at smaller times but later on the curve flattens and the star-formation rate at the pericenter is almost identical to the low velocity case SF90. On the other hand the star formation generated by a compression perpendicular to the LMC disk increases

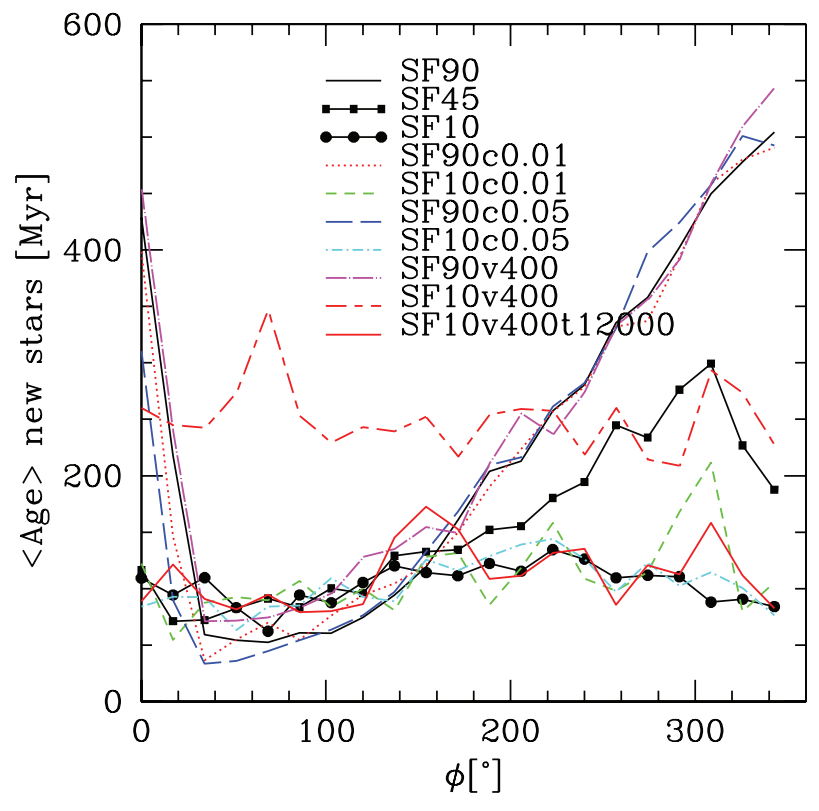

Figure 10 External disk mean stellar age in function of the azimuthal angle $\phi$ for a selection of the runs listed in Figure 1. The youngest stars are located at $30<\phi<100^{\circ}$.

with increasing ram-pressure values (it shows a decrement only at the end of the simulation) and in the case of the high velocity run SF10v4000 reaches a peak $\sim 1.5$ times higher than in SF10.

By introducing a temperature floor well above the cutoff in the cooling function (heating sources and turbulent motions in the gas are neglected in our simple cooling model) we prevent local instabilities in the gaseous disk and we suppress star formation affecting both the threshold in external pressure and the star-formation rate peak (SF10v400t12000 and SF10v400t15000).

Finally, a hot halo ten times less dense than the one assumed in our favourite model would reduce drastically star formation in a face-on LMC and suppress completely the star formation on the leading edge of the disk.

Figure 10 represents the mean stellar age of the external disk versus the azimuthal angle $\phi$ for selected runs in Table 1. The maximum increment in age in a clockwise direction is associated to edge-on runs, with the youngest stars located at $30<\phi<100^{\circ}$. Clearly the gradient in age is much less visible in runs with $i<90^{\circ}$.

\section{Conclusions}

The compression of the leading edge of the LMC disk by a low-density ionized halo can account for the high density $\mathrm{HI}$ region and the intense star formation activity that characterize the eastern border of the disk. The combined effect of ram-pressure and rotation can also explain the progression in age of the star forming regions along the outer east and north edge of the LMC. At the same time the compression directed perpendicularly to the disk produces local instabilities in the external gas distribution and a clumpy structure characterized by voids and high density filaments. The star formation events associated with 
these high density structures could explain the delocalized star formation activity observed across the entire disk of the LMC.

\section{Acknowledgments}

The numerical simulations were performed on the zBox 1 supercomputer at the University of Zurich and on the local SGI-Altix $3700 \mathrm{Bx} 2$ (partly funded by the cluster of excellence 'Origin and Structure of the Universe'). This work was partly supported by the DFG Sonderforschungsbereich 375 'Astro-Teilchenphysik'.

\section{References}

Bekki, K. \& Chiba, M., 2005, MNRAS, 356, 680

Bekki, K. \& Chiba, M., 2007, MNRAS, 381, L16

Bland-Hawthorn, J., Sutherland, R., Agertz, O. \& Moore, B., 2007, ApJ, 670, L109

Blondiau, M. J., Kerp, J., Mebold, U. \& Klein, U., 1997, A\&A, 323, 585

Braun, J. M., Bomans, D. J., Will, J.-M. \& de Boer, K. S., 1997, A\&A, 328, 167

Cioni, M.-R. L., Girardi, L., Marigo, P. \& Habing, H. J., 2006, A\&A, 448,77 de Boer, K. S., Braun, J. M., Vallenari, A. \& Mebold, U., 1998, A\&A, 329, L49

Gerola, H. \& Seiden, P. E., 1978, ApJ, 223, 129

Grebel, E. K. \& Brandner, W., 1998, in The Magellanic Clouds and Other Dwarf Galaxies, Eds. Richtler, T. \& Braun, J. M. (Aachen: Shaker Verlag), 151

Hernquist, L., 1993, ApJ, 86, 389

Kallivayalil, N., van der Marel, R. P., Alcock, C., Axelrod, T., Cook, K. H., Drake, A. J. \& Geha, M., 2006, ApJ, 638, 772

Katz, N., 1992, ApJ, 391, 502

Katz, N., Weinberg, D. H. \& Hernquist, L., 1996, ApJS, 105, 19

Kennicutt, R. C., Jr., 1989, ApJ, 344, 685

Mastropietro, C., Moore, B., Mayer, L., Wadsley, J. \& Stadel, J., 2005, MNRAS, 363, 509

Pedersen, K., Rasmussen, J., Sommer-Larsen, J., Toft, S., Benson, A. J. \& Bower, R. G., 2006, NewA, 11, 465

Roediger, E. \& Brüggen, M., 2006, MNRAS, 369, 567

Sandage, A., 1986, A\&A, 161, 89

Sembach, K. R. et al., 2003, ApJS, 146, 165

Staveley-Smith, L., Kim, S., Calabretta, M. R., Haynes, R. F. \& Kesteven, M. J., 2003, MNRAS, 339, 87

Toomre, A., 1964, ApJ, 139, 1217

van der Marel, R. P., Alves, D. R., Hardy, E. \& Suntzeff, N. B., 2002, AJ, 124, 2639

Wadsley, J. W., Stadel, J. \& Quinn, T., 2004, NewA, 9, 137 